\title{
ON THE INTEGRABILITY OF COMPLEX TRIGONOMETRIC SERIES
}

\author{
WILLIAM O. BRAY AND VERA B. STANOJEVIĆ
}

\begin{abstract}
The classical criterion for the Fourier character of cosine series and sine series is generalized to complex trigonometric series. Our treatment unifies the ideas applied to cosine and sine series. A notion of complex convex sequences is developed generalizing a result of Young [1] concerning the integrability of cosine series.
\end{abstract}

1. Introduction. The problem of determining the Fourier character of the cosine series

$$
\frac{a(0)}{2}+\sum_{n=1}^{\infty} a(n) \cos n x
$$

classically was approached via the de la Vallé Poisson theorem. There are two difficulties in applying this result: the difficulty of pointwise convergence of (1.1) except on a denumerable set, and the difficulty in verifying the integrability of the sum function. In a series of classical and later results, those difficulties were overcome by assuming various kinds of regularity and/or speed conditions on the coefficients $\{a(n)\}$. All these assumptions imply that the sequence $\{a(n)\}$ is of bounded variation $\left(\sum_{n=0}^{\infty}|\Delta a(n)|<\infty, \Delta a(n)=a(n)-a(n+1)\right)$, which further implies the existence of the pointwise limit $f(x)$ of $(1.1)$ on $(0, \pi]$. Hence the difficult side of the problem is to verify the integrability of $f$.

To exemplify, we cite the following classical sufficient conditions for $f \in L^{1}(0, \pi]$ : Young [1]- $\{a(n)\}$ is convex $\left(\Delta^{2} a(n) \geqslant 0\right.$ for all $\left.n\right)$; Kolmogorov [2]- $\{a(n)\}$ is quasi-convex $\left(\sum_{n=0}^{\infty}(n+1)\left|\Delta^{2} a(n)\right|<\infty\right)$; Sidon [3], whose condition was reformulated in a more succinct form by Telyakovskii [4] $-\{a(n)\}$ belongs to the class $S$ (there exists a nonincreasing sequence $\{A(n)\}_{0}^{\infty}$ such that $\sum_{n=0}^{\infty} A(n)<\infty$ and $|\Delta a(n)| \leqslant A(n)$ for all $n)$. Analogous results hold (see e.g., [4]) for the conjugate series or sine series

$$
\sum_{n=1}^{\infty} a(n) \sin n x
$$

whose pointwise limit on $(0, \pi]$ is denoted $g(x)$.

Received by the editors February 20, 1984. Presented to the Society at the 90th annual meeting in Louisville, Kentucky, January 1984. Also presented at Workshop in Analysis and its Applications, June 10-20, 1984; VUK, Yugoslavia.

1980 Mathematics Subject Classification. Primary 42A20, 42A32.

Key words and phrases. Integrability of trigonometric series, $L^{1}$-convergence of Fourier series, complex monotone sequences, complex convex sequences. 
The aforementioned results are corollaries of the following recent result due to Fomin [5].

THEOREM. Let $a(n)=o(1)(n \rightarrow \infty)$, and for some $p>1$ let

$$
\sum_{n=1}^{\infty}\left(\frac{\sum_{k=n}^{\infty}|\Delta a(k)|^{p}}{n}\right)^{1 / p}<\infty \text {. }
$$

Then (i) $f \in L^{1}(0, \pi]$, and

(ii) $g \in L^{1}(0, \pi]$ if and only if $\sum_{n=1}^{\infty}|a(n)| / n<\infty$.

The purpose of this paper is to generalize Fomin's result and its corollaries to complex trigonometric series

$$
\sum_{|n|<\infty} c(n) e^{i n t}
$$

where $\{c(n)\}_{|n|<\infty} \subset \mathbf{C}$ and $t \in T=\mathbf{R} / 2 \pi Z$. Our results (§3) serve to unify classical ideas applied separately to cosine and sine series, and the method of proof provides greater insight into the nature of (1.3) as an integrability condition, as well as to provide possibilities for further generalization. Certain difficulties are encountered in our generalization due to the nature of possible gaps in the sequence $\{|c(n)-c(-n)|\}$. These are overcome in $\$ 3$ with the notion of intrinsically odd sequences.

2. Definitions and lemmas. In this section certain lemmas and definitions are given which are convenient and/or intrinsic to subsequent argument.

Recall that an increasing sequence of positive integers $\left\{m_{n}\right\}_{0}^{\infty}$ is lacunary if, for some $r>1, m_{n+1} / m_{n} \geqslant r$ for all $n$. An essential property of lacunary sequences is that $\sum_{k=n}^{\infty} 1 / m_{k}=O\left(1 / m_{n}\right)(n \rightarrow \infty)$. A lacunary sequence of positive integers is said to be $O$-lacunary if, for some $R>1, m_{n+1} / m_{n} \leqslant R$ for all $n$. Note that a O-lacunary sequence satisfies $m_{n+1}-m_{n}=O\left(m_{n}\right)(n \rightarrow \infty)$. Without loss of generality in the sequel, we suppose $m_{0}=1$.

The notion of O-lacunary plays an essential role through the following easily proven lemma.

LEMMA 2.1. Let $\{a(n)\}_{1}^{\infty}$ be a positive sequence of bounded variation. Then the series $\sum_{n=1}^{\infty} a(n) / n$ and $\sum_{n=0}^{\infty} a\left(m_{n}\right)$ are equiconvergent for any $O$-lacunary sequence $\left\{m_{n}\right\}_{0}^{\infty}$.

In [6] a notion of complex monotone sequences are introduced, i.e. the sequence $\{c(n)\}_{1}^{\infty} \subset C$ is said to be complex monotone if there exists a cone $K\left(\theta_{0}\right)=\{z \in C \mid$ $\left.|\arg z| \leqslant \theta_{0}<\pi / 2\right\}$ such that $\Delta c(n) \in K\left(\theta_{0}\right)$ for all $n$. It was further shown that a complex monotone sequence is of bounded variation. Following these ideas, a sequence $\{c(n)\}_{|n|<\infty} \subset C$ is said to be complex convex if there exists a cone $K\left(\theta_{0}\right)$ such that $\operatorname{sgn}(n) \Delta^{2} c(n) \in K\left(\theta_{0}\right)$ for $|n|>1$. It is not hard to see that the null sequences $\{c(n)\}_{1}^{\infty}$ and $\{c(-n)\}_{1}^{\infty}$ (or their negatives) are complex monotone. Summarizing, we have the following.

LEMMA 2.2. Let $\{c(n)\}_{|n|<\infty} \subset C$ be a complex convex null sequence. Then $\sum_{|n|<\infty}|\Delta c(n)|<\infty$, i.e. $\{c(n)\}$ is in the class BV. 
LEMMA 2.3. Let $c(n)=o(1) \quad(|n| \rightarrow \infty)$ and let $\{c(n)\}_{|n|<\infty} \in \mathrm{BV}$. Then $\lim _{n \rightarrow \infty} S_{n}(t)=f(t)$ exists on $T-\{0\}$, where

$$
S_{n}(t)=\sum_{|k| \leqslant n} c(k) e^{i k t}
$$

Proof. We apply an identity given in [7], i.e. setting $\omega(t)=1-e^{-i t}$, then

$$
S_{n}(t)=\frac{1}{\omega(t)} \sum_{k=-n-1}^{n-1} \Delta c(k) e^{i k t}-\left[\frac{c(-n-1) e^{-i(n+1) t}-c(n) e^{i n t}}{\omega(t)}\right],
$$

from which the lemma follows. In fact the pointwise limit on $T-\{0\}$ is given by

$$
f(t)=\sum_{|n|<\infty} \Delta c(k) \frac{e^{i k t}}{\omega(t)} .
$$

In particular, the conclusion of the lemma holds if $\{c(n)\}_{|n|<\infty}$ is a null complex convex sequence.

Finally, a null sequence $\{c(n)\}_{|n|<\infty} \subset C$ is said to be quasi-convex if

$$
\sum_{|n|<\infty}(|n|+1)\left|\Delta^{2} c(n)\right|<\infty .
$$

The relation between complex convex sequences and quasi-convex sequences is as follows.

LEMMA 2.4. Let $\{c(n)\}_{|n|<\infty}$ be a null complex convex sequence. Then $\{c(n)\}_{|n|<\infty}$ is quasi-convex if and only if

$$
|n||\Delta c(n)|=O(1) \quad(|n| \rightarrow \infty) .
$$

Proof. For necessity we have

$$
\sum_{|k|=n}^{\infty} k\left|\Delta^{2} c(k)\right| \geqslant n \sum_{|k|=n}^{\infty}\left|\Delta^{2} c(k)\right| \geqslant n[|\Delta c(n)|+|\Delta c(-(n-1))|],
$$

from which (2.2) follows. For sufficiency we apply an inequality of M. Petrović [8]:

$$
\begin{aligned}
\sum_{k=1}^{n} k\left|\Delta^{2} c(k)\right| & \leqslant \frac{1}{\cos \theta_{0}}\left|\sum_{k=1}^{n} k \Delta^{2} c(k)\right| \\
& =\frac{1}{\cos \theta_{0}}\left|\sum_{k=0}^{n-1} \Delta c(k+1)-n \Delta c(n+1)\right| \\
& \leqslant \frac{1}{\cos \theta_{0}}[|c(1)|+|c(n+1)|+(n+1)|\Delta c(n+1)|],
\end{aligned}
$$

from which it follows that $\sum_{k=1}^{\infty} k\left|\Delta^{2} c(k)\right|<\infty$. In a similar fashion, $\sum_{k=-\infty}^{-1}|k|\left|\Delta^{2} c(k)\right|<\infty$, concluding the proof.

3. Results. A sequence $\{c(n)\}_{|n|<\infty} \subset C$ is said to be intrinsically odd if for some O-lacunary sequence $\left\{m_{n}\right\}_{n=0}^{\infty}$, we have $\left|c\left(m_{n}\right)-c\left(m_{-n}\right)\right|>0$ for all $n$. This notion is fundamental in our method for handling the integrability of complex 
trigonometric series; in the case of real sine series the condition causes no loss of generality.

THEOREM 1. Let $\{c(n)\}_{|n|<\infty} \subset C$ be a null intrinsically odd sequence and, for some $p>1$, let

$$
\sum_{n=1}^{\infty}\left(\frac{\sum_{|k|=n}^{\infty}|\Delta c(k)|^{p}}{n}\right)^{1 / p}<\infty .
$$

Then (i) $\lim _{n \rightarrow \infty} S_{n}(t)=f(t)$ exists on $T-\{0\}$, and (ii) $f \in L^{1}(T)$ if and only if $\sum_{n=1}^{\infty}|c(n)-c(-n)| / n<\infty$.

Proof. Using the Cauchy condensation test and the method of [9], one can show that the series in (3.1) is equiconvergent with

$$
\sum_{n=0}^{\infty} 2^{n}\left(\frac{1}{2^{n}} \sum_{|k|=2^{n}}^{2^{n+1}-1}|\Delta c(k)|^{p}\right)^{1 / p} .
$$

Consequently, (3.1) implies $\{c(n)\}_{|n|<\infty} \in \mathrm{BV}$ by Jensen's inequality; this proves (i) by Lemma 2.3. Without loss of generality (by (3.2)), we may assume $1<p \leqslant 2$. For (ii) our primary tool is the Hölder-Hausdorff-Young technique used in prior work (e.g. $[10,11])$. Let $\left\{m_{n}\right\}_{0}^{\infty}$ be a O-lacunary sequence such that $\left|c\left(m_{n}\right)-c\left(-m_{n}\right)\right|>0$ for all $n$, and for $N \geqslant 1$, let $U_{N}=\left[-\pi,-\pi / m_{N+1}\right) \cup\left(\pi / m_{N+1}, \pi\right]$. The proof will be concluded via the following estimate: for $1<p \leqslant 2$ and uniformly in $N=$ $1,2, \ldots$,

$$
\begin{aligned}
\int_{U_{N}}|f(t)| d t= & B_{N} \sum_{n=0}^{N}\left|c\left(m_{n}\right)-c\left(-m_{n}\right)\right| \\
& +O\left(\sum_{n=0}^{\infty} m_{n}\left(\frac{1}{m_{n}} \sum_{|k|=m_{n}}^{\infty}|\Delta c(k)|^{p}\right)^{1 / p}\right),
\end{aligned}
$$

where $\left\{B_{N}\right\}_{0}^{\infty}$ is a bounded sequence of positive numbers such that $B_{N} \geqslant \alpha>0$ for all $N$. Using a generalized Cauchy condensation test, the series in the $O$-term of (3.3) is equiconvergent with (3.1). The proof is concluded by Lemma 2.1 provided we verify (3.3). For $n \geqslant 0$ let

$$
T_{n}=\left[-\frac{\pi}{m_{n}},-\frac{\pi}{m_{n+1}}\right) \cup\left(\frac{\pi}{m_{n+1}}, \frac{\pi}{m_{n}}\right]
$$

then using (2.1), for $N=1,2, \ldots$,

$$
\begin{aligned}
& \left|\int_{U_{N}}\right| f(t)\left|d t-\sum_{n=0}^{N} \int_{T_{n}}\right| \sum_{k=-m_{n}}^{m_{n}-1} \Delta c(k) \frac{e^{i k t}}{\omega(t)}|d t| \\
& \quad \leqslant \sum_{n=0}^{N} \int_{T_{n}}\left|\sum_{|k| \geqslant m_{n}} \Delta c(k) \frac{e^{i k t}}{\omega(t)}\right| d t+\sum_{n=0}^{N}\left|\Delta c\left(-m_{n}\right)\right| \int_{T_{n}} \frac{d t}{|\omega(t)|} .
\end{aligned}
$$


Denote the first term on the right-hand side of (3.4) by $Q_{N}$ and note that $|\omega(t)|=2|\sin (t / 2)|$. Applying the inequalities of Hölder and Hausdorff-Young, one obtains the estimate

$$
\begin{aligned}
Q_{N} & \leqslant \sum_{n=0}^{N}\left(\int_{T_{n}} \frac{d t}{|\omega(t)|^{p}}\right)^{1 / p}\left\|\sum_{|k|=m_{n}} \Delta c(k) e^{i k t}\right\|_{q} \\
& \leqslant A_{p} \sum_{n=0}^{\infty} m_{n}^{1-1 / p}\left(\sum_{|k|=m_{n}}^{\infty}|\Delta c(k)|^{p}\right)^{1 / p},
\end{aligned}
$$

where $\|\cdot\|_{q}$ denotes the $L^{q}(T)$-norm $(1 / p+1 / q=1)$ and $A_{p}$ is a constant dependent only on $p$. The second term on the right-hand side of (3.4) is majorized by

$$
\pi \sum_{n=0}^{N}\left|\Delta c\left(-m_{n}\right)\right| \lg \frac{m_{n+1}}{m_{n}} \leqslant \pi \lg R \sum_{n=0}^{\infty}|\Delta c(n)|,
$$

where $R>1$ is such that $m_{n+1} / m_{n} \leqslant R$ for all $n$. Using Jensen-inequality considerations as before, the latter estimate may be combined with that for $Q_{N}$; hence for $N=1,2, \ldots$,

$$
\begin{aligned}
\int_{U_{N}}|f(t)| d t= & \sum_{n=0}^{N} \int_{T_{n}}\left|\sum_{k=-m_{n}}^{m_{n}-1} \Delta c(k) e^{i k t}\right| \frac{d t}{|\omega(t)|} \\
& +O\left(\sum_{n=0}^{\infty} m_{n}\left(\frac{1}{m_{n}} \sum_{|k|=m_{n}}^{\infty}|\Delta c(k)|^{p}\right)^{1 / p}\right)
\end{aligned}
$$

Denote the first term on the right-hand side of (3.5) by $Q_{N}^{\prime}$. Using power series the following estimate is easily obtained: for all $k \in Z$,

$$
\left|\frac{e^{i k t}}{|\omega(t)|}-\frac{1}{2|\sin (t / 2)|}\right| \leqslant A|k|
$$

uniformly on $T$, where $A$ is an absolute constant. Hence,

$$
\begin{aligned}
\left|Q_{N}^{\prime}-\frac{1}{2} \sum_{n=0}^{N} \int_{T_{n}}\right| \sum_{k=-m_{n}}^{m_{n}-1} \Delta c(k)\left|\frac{d t}{|\sin (t / 2)|}\right| & \leqslant A \sum_{n=0}^{N} \int_{T_{n}} \sum_{|k| \leqslant m_{n}}|k||\Delta c(k)| d t \\
& \leqslant A \sum_{n=0}^{N} \frac{1}{m_{n}} \sum_{|k| \leqslant m_{n}}|k||\Delta c(k)|,
\end{aligned}
$$

where in the last inequality all absolute constants have been absorbed into $A$. Let $r>1$ be the largest number such that $m_{n+1} / m_{n} \geqslant r$ for all $n$. Then interchanging the order of summation,

$$
\begin{aligned}
\sum_{n=0}^{N} \frac{1}{m_{n}} \sum_{|k| \leqslant m_{n}}|k||\Delta c(k)| & =\sum_{|k| \leqslant m_{N}}|k||\Delta c(k)| \sum_{\substack{l \\
|k| \leqslant m_{l}}}^{N} \frac{1}{m_{l}} \\
& \leqslant \sum_{|k| \leqslant m_{N}}|k||\Delta c(k)| \sum_{l \geqslant\left[\mathrm{lg}_{R}|k|\right]}^{\infty} \frac{1}{m_{l}},
\end{aligned}
$$


where $\left[\lg _{R}|k|\right]$ is the greatest integer in the logarithm to base $R$ of $|k|$. Since

$$
\sum_{l>\left[\mathrm{lg}_{R}|k|\right]} \frac{1}{m_{l}} \leqslant A^{\prime} \frac{1}{m_{\left[\mathrm{g}_{R}|k|\right]}} \leqslant \frac{A^{\prime}}{|k| \lg _{R} r},
$$

where $A^{\prime}$ is an absolute constant, we have

$$
\sum_{n=0}^{N} \frac{1}{m_{n}} \sum_{|k| \leqslant m_{n}}|k||\Delta c(k)| \leqslant \frac{A^{\prime}}{\lg _{R} r} \sum_{|k| \leqslant m_{N}}|\Delta c(k)| .
$$

Consequently, uniformly in $N$,

$$
Q_{N}^{\prime}=\frac{1}{2} \sum_{n=0}^{N}\left|c\left(m_{n}\right)-c\left(-m_{n}\right)\right| \int_{T_{n}} \frac{d t}{|\sin (t / 2)|}+O\left(\sum_{|k|<\infty}|\Delta c(k)|\right) .
$$

Since $|1 / 2| \sin (t / 2)|-1 /| t||$ is uniformly bounded on $T$, we get

$$
\begin{aligned}
Q_{N}^{\prime} & =\sum_{n=0}^{N}\left|c\left(m_{n}\right)-c\left(-m_{n}\right)\right| \int_{T_{n}} \frac{d t}{|t|}+O\left(\sum_{|k|<\infty}|\Delta c(k)|\right) \\
& =\sum_{n=0}^{N}\left|c\left(m_{n}\right)-c\left(-m_{n}\right)\right| \lg \frac{m_{n+1}}{m_{n}}+O\left(\sum_{|k|<\infty}|\Delta c(k)|\right),
\end{aligned}
$$

again uniformly in $N$. Because $\lg r \leqslant \lg \left(m_{n+1} / m_{n}\right) \leqslant \lg R$, we have

$$
Q_{N}^{\prime}=B_{N} \sum_{n=0}^{N}\left|c\left(m_{n}\right)-c\left(-m_{n}\right)\right|+O\left(\sum_{|k|<\infty}|\Delta c(k)|\right)
$$

for an appropriate bounded sequence $B_{N}$, with $B_{N} \geqslant \alpha>0$ for all $n$. Again applying aforementioned Jensen-inequality techniques, the $O$-term in the estimate for $Q_{N}^{\prime}$ may be combined with our estimate for $Q_{N}$. This completes the proof of Theorem 1 .

Notice that the above result does not apply when $\{c(n)\}_{|n|<\infty}$ is an even sequence $(c(n)=c(-n)$ for all $n)$. In this case we have the following result, easily deduced using the above techniques.

THEOREM 2. Let $\{c(n)\}_{|n|<\infty} \subset \mathrm{C}$ be an even null sequence and, for some $p>1$, let (3.1) hold. Then (i) $\lim _{n \rightarrow \infty} S_{n}(t)=f(t)$ exists for $t \in T-\{0\}$, and (ii) $f \in L^{1}(T)$.

Three corollaries of classical origin can now be proved; as these are all based on strengthening (3.1), they are stated for intrinsically odd sequences only.

The Telyakovskii-Sidon class $S$ is extended to complex sequences as follows: a null sequence $\{c(n)\}_{|n|<\infty} \subset \mathbf{C}$ is said to belong to the class $S_{c}$ if there exists a nonincreasing sequence $\{A(n)\}_{0}^{\infty}$ such that $\sum_{n=1}^{\infty} A(n)<\infty$ and

$$
\max \{|\Delta c(n)|,|\Delta c(-n)|\} \leqslant A_{n} \text { for all } n \geqslant 1
$$

Corollary 1.1. Let $\{c(n)\}_{|n|<\infty} \in S_{c}$ and intrinsically odd. Then (i) and (ii) of Theorem 1 hold. 
Proof. Since the series in (3.1) is equiconvergent with (3.2), it suffices to prove convergence of (3.2). In fact, for all $N$,

$$
\begin{aligned}
\sum_{n=0}^{N} 2^{n}\left(\frac{1}{2^{n}} \sum_{|k|=2^{n}}^{2^{n+1}-1}|\Delta c(k)|^{p}\right)^{1 / p} & \leqslant 2^{1 / p} \sum_{n=0}^{N} 2^{n}\left(\frac{1}{2^{n}} \sum_{k=2^{n}}^{2^{n+1}-1} A(k)^{p}\right)^{1 / p} \\
& \leqslant 2^{1 / p} \sum^{\infty} 2^{n} A\left(2^{n}\right) .
\end{aligned}
$$

The last series converges since it is equiconvergent with $\sum_{n=0}^{\infty} A(n)$.

COROllary 1.2. Let $\{c(n)\}_{|n|<\infty} \subset C$ be a quasi-convex intrinsically odd null sequence. Then (i) and (ii) of Theorem 1 hold.

COROLlaRY 1.3. Let $\{c(n)\}_{|n|<\infty}$ be a null complex convex intrinsically odd sequence and let $|n||\Delta c(n)|=O(1)(|n| \rightarrow \infty)$. Then (i) and (ii) of Theorem 1 hold.

From Theorem 1 (or Theorem 2) one easily obtains the following result concerning the integrability of the conjugate series $\Sigma_{|n|<\infty}-i \operatorname{sgn}(n) c(n) e^{i n t}$. The pointwise limit of this series on $T-\{0\}$ is denoted $\tilde{f}(t)$.

Corollary 1.4. Let $\{c(n)\}_{|n|<\infty}$ and $\{-i \operatorname{sgn}(n) c(n)\}_{|n|<\infty}$ be intrinsically odd, and for some $p>1$ let (3.1) hold. Then $f, \tilde{f} \in L^{1}(T)$ if and only if $\Sigma_{|n|<\infty}|c(n)| / n<\infty$.

Note that the condition " $\{-i \operatorname{sgn}(n) c(n)\}$ be intrinsically odd" says that $\mid c\left(m_{n}\right)$ $+c\left(-m_{n}\right) \mid>0$ for some O-lacunary sequence $\left\{m_{n}\right\}_{0}^{\infty}$. An analogous corollary holds for Theorem 2 as well as corollaries involving class $S_{c}$, quasi-convexity and convexity.

4. $L^{1}$-convergence and additional remarks. Let $f \in L^{1}(T)$ and, for some $1<p \leqslant 2$, let

$$
\lim _{\lambda \downarrow 1} \lim _{n \rightarrow \infty} \sum_{|k|=n}^{[\lambda n]}|k|^{p-1}|\Delta \hat{f}(k)|^{p}=0 .
$$

Then in [11] it was shown that $\left\|S_{n}(f)-f\right\|=o(1)(n \rightarrow \infty)$ if and only if $\hat{f}(n) \lg (n)$ $=o(1)(|n| \rightarrow \infty)$. As (3.1) implies (4.1), we obtain the following result.

COROLlaRY. Let $\{c(n)\}_{|n|<\infty}$ be an intrinsically odd null sequence and, for some $p>1$, let (3.1) hold. Then $\left\|S_{n}-f\right\|=o(1)(n \rightarrow \infty)$ if and only if

$$
\sum_{n=1}^{\infty} \frac{|c(n)-c(-n)|}{n}<\infty \quad \text { and } c(n) \lg |n|=o(1) \quad(|n| \rightarrow \infty)
$$

The numerous variants and corollaries to Theorem 3.1 can also be developed in this vein.

In contrast with real cosine series (or sine series) with convex coefficients, in the case of complex trigonometric series with complex convex coefficients one needs the further condition

$$
|n||\Delta c(n)|=O(1) \quad(|n| \rightarrow \infty)
$$


to achieve an integrability result. Condition $(*)$ is for free in the real case. This suggests a deeper study of complex convexity in relation to the integrability problem in an attempt to weaken $(*)$.

Finally, the work in [6], along with our notion of complex convexity, suggests that many results involving regularity conditions of monotonicity type for sine and cosine series (see [12] for other examples) can be extended to complex trigonometric series.

\section{REFERENCES}

1. W. H. Young, On the Fourier series of bounded functions, Proc. London Math. Soc. (2) 12 (1913).

2. A. N. Kolmogorov, Sur l'ordre de grandeur des coefficients de la séries de Fourier-Lebesgue, Bull. Acad. Polon. Sci. Math. Astronom. Phys. (1923).

3. S. Sidon, Hinreichende Bedingungen für den Fourier Charakter einer Trigonometrischen Reiche, J. London Math. Soc. (2) 14 (1939).

4. S. A. Telyakovskii, On a sufficient condition of Sidon for the integrability of trigonometric series, Math. Notes 14 (1973).

5. G. A. Fomin, A class of trigonometric series, Math. Notes 23 (1978).

6. V. B. Stanojević, $L^{1}$-convergence of Fourier series with complex quasi-monotone coefficients, Proc. Amer. Math. Soc. 86 (1982), 241-247.

7. __ On a theorem of P. L. Uljanov, Proc. Amer. Math. Soc. 90 (1984), 370-372.

8. M. Petrović, Théorème sur les intégrales curvilignes, Publ. Math. Univ. Beograd. II (1933).

9. W. O. Bray, On a theorem of Stanojević, Proc. Amer. Math. Soc. 83 (1981), 59-62.

10. W. O. Bray and Č. V. Stanojević, Tauberian $L^{1}$-convergence classes of Fourier series. I, Trans. Amer. Math. Soc. 275 (1983), 59-69.

11. Tauberian $L^{1}$-convergence classes of Fourier series. II, Math. Ann. (to appear).

12. R. P. Boas, Integrability theorems for trigonometric transforms, Springer-Verlag, 1965.

Department of Mathematics, University of Maine, Orono, Maine 04469

Department of Mathematics, University of MisSOURI, Rolla, MisSOURI 65401 